\title{
Common fixed point theorems in Menger probabilistic met- ric spaces using the CLRg property
}

\author{
Shuang Wang ${ }^{\mathrm{a}}$, Dingbian Qian ${ }^{\mathrm{b}, *}$ \\ a School of Mathematical Sciences, Yancheng Teachers University, Yancheng, 224051, Jiangsu, P. R. China. \\ ${ }^{b}$ School of Mathematical Sciences, Soochow University, Suzhou 215006, China.
}

\begin{abstract}
Under some weaker conditions of the $\varphi$, some common fixed point theorems for weakly compatible mappings are established in Menger probabilistic metric spaces. Using the CLRg property, our results show that the completeness of underlying spaces is not necessary for fixed point theorems. In order to illustrate our results, we provide two examples in which other theorems cannot be applied.
\end{abstract}

Keywords: Menger probabilistic metric spaces, weakly compatible mappings, common fixed points, common limit in the range property.

2010 MSC: 47H10, 54H25.

(C)2019 All rights reserved.

\section{Introduction}

Fixed point theorem for probabilistic k-contraction was first studied in 1972 by Sehgal and BharuchaReid [14]. This is a probabilistic version of the classical Banach Contraction Principle. Later on, many authors obtained some fixed point theorems for probabilistic $\varphi$-contraction, which is a natural generalization of probabilistic k-contraction. But, many results are obtained under the assumption that $\varphi$ satisfies $\sum_{n=1}^{\infty} \varphi^{n}(t)<\infty$ for all $t>0$ and some other conditions (see, e.g. [3, 6, 7, 11]). Ćirić [2] has pointed out, the condition $\sum_{n=1}^{\infty} \varphi^{n}(t)<\infty$ for all $t>0$ is very strong and difficult for testing in practice. In [2], Ćirić improved the condition by introducing condition (CBW): $\varphi(0)=0, \varphi(t)<t$ and $\liminf _{r \rightarrow t^{+}} \varphi(t)<t$ for all $t>0$. Later, Jachymski [8] presented the condition (a): $0<\varphi(t)<t$ and $\lim _{n \rightarrow \infty} \varphi^{n}(t)=0$ for all $t>0$. In order to weaken the condition (a) further, Fang [4] introduced the condition (b): for each $t>0$ there exists $r \geqslant t$ such that $\lim _{n \rightarrow \infty} \varphi^{n}(r)=0$ in the context of Menger probabilistic metric spaces and fuzzy metric spaces. The condition (b) is a quite weak condition.

However, the results in $[2,4,8]$ need the completeness of underlying spaces. Under the CLRg property, it is not necessary to assume the completeness of the spaces, which is an important advantage compared

\footnotetext{
*Corresponding author

Email addresses: wangshuang19841119@163.com (Shuang Wang), dbqian@suda.edu .cn (Dingbian Qian)

doi: $10.22436 /$ jnsa.012.01.05
}

Received: 2017-10-07 Revised: 2018-08-05 Accepted: 2018-08-17 
with the most of theorems in fixed point theory. For example, Sintunavarat and Kumam [15] coined the idea of common limit in the range property (called CLRg) in an usual metric space in 2011. In 2012, Jain et al. [9] extended the concept of CLRg property in the coupled case and also established a common fixed point theorem for weakly compatible mappings in fuzzy metric spaces. Most recently, Hierro and Sintunavarat [12] generalized the results in [9] by using the generalized contractive conditions and the CLRg property in fuzzy metric spaces. We also remind the reader of the following facts: (i) results in [9] are obtained under the condition $\sum_{n=1}^{\infty} \varphi^{n}(t)<\infty$ for all $t>0$; (ii) Hierro and Sintunavarat [12] obtained the common fixed point for weakly compatible mappings under the condition $\varphi(t)>0$ and $\lim _{n \rightarrow \infty} \varphi^{n}(t)=0$ for all $\mathrm{t}>0$.

Motivated and inspired by results of papers [2, 4, 8, 9, 12, 14, 15], we establish some new common fixed point theorems for weakly compatible mappings in Menger probabilistic metric spaces by using the CLRg property and the condition (b). Our results show that the completeness of Menger probabilistic metric spaces is not necessary for fixed point theorems. In order to illustrate our results, we provide two examples in which other theorems cannot be applied.

\section{Preliminaries}

In order to fix the framework needed to state our main results, we recall the following notions.

Throughout this paper, let $\mathbb{I}=[0,1]$ subset of $\mathbb{R}, \mathbb{R}=(-\infty,+\infty), \mathbb{R}^{+}=[0,+\infty)$, and $\mathbb{N}$ be the set of all natural numbers. For brevity, $f(x)$ and $g(x)$ will be denoted by $f x$ and $g x$, respectively. Let $\Phi$ denote the family of all functions $\varphi: \mathbb{R}^{+} \rightarrow \mathbb{R}^{+}$verifying the condition (c): $\varphi(t)>0$ and $\lim _{n \rightarrow \infty} \varphi^{n}(t)=0$ for all $t>0$, and let $\Phi_{w}$ denote the class of all functions $\varphi: \mathbb{R}^{+} \rightarrow \mathbb{R}^{+}$satisfying the condition (b), i.e., for each $t>0$ there exists $r \geqslant t$ such that $\lim _{n \rightarrow \infty} \varphi^{n}(r)=0$.

Obviously, the condition $\lim _{n \rightarrow \infty} \varphi^{n}(t)=0$ for all $t>0$ implies the condition (b). The following example shows that the reverse is not true in general. Hence $\Phi \subseteq \Phi_{w}$.

Example 2.1 ([4]). Let the function $\varphi: \mathbb{R}^{+} \rightarrow \mathbb{R}^{+}$be defined by

$$
\varphi(t)= \begin{cases}\frac{t}{1+t}, & \text { if } 0 \leqslant t<1 \\ -\frac{t}{3}+\frac{4}{3}, & \text { if } 1 \leqslant t \leqslant 2 \\ t-\frac{4}{3}, & \text { if } 2<t<\infty\end{cases}
$$

Notice that $\varphi \in \Phi_{w}$ but $\varphi \notin \Phi$.

Lemma 2.2 ([4]). Let $\varphi \in \Phi_{w}$, then for each $\mathrm{t}>0$ there exists $r \geqslant t$ such that $\varphi(r)<t$.

A mapping $F: \mathbb{R} \rightarrow \mathbb{R}^{+}$is called a distribution if it is non-decreasing left-continuous with $\sup _{t \in \mathbb{R}} F(t)=$ 1 and $\inf _{t \in \mathbb{R}} F(t)=0$. The set of all distribution functions is denoted by $\mathbb{D}$, and $\mathbb{D}^{+}=\{F \mid F \in \mathbb{D}, F(0)=0\}$. A special element $\mathrm{H}$ of $\mathbb{D}^{+}$is defined by

$$
H(t)= \begin{cases}0, & \text { if } t \leqslant 0 \\ 1, & \text { if } t>0\end{cases}
$$

A mapping $f: X \rightarrow X$ is called a probabilistic $k$-contraction if it satisfies $F_{f x, f y}(k t) \geqslant F_{x, y}(t)$ for all $x, y \in X$ and $t>0$, where $k \in(0,1)$. A mapping $f: X \rightarrow X$ is called a probabilistic $\varphi$-contraction if it satisfies $F_{f x, f y}(\varphi(t)) \geqslant F_{x, y}(t)$ for all $x, y \in X$ and $t>0$.

Definition 2.3 ([13]). A triangular norm (also called a t-norm) is a map $*: \mathbb{I}^{2} \rightarrow \mathbb{I}$ that is associative, commutative, non-decreasing in both arguments and has 1 as identity. A t-norm is continuous if it is continuous in $\mathbb{I}^{2}$ as mapping. If $a_{1}, a_{2}, \ldots, a_{m} \in \mathbb{I}$, then

$$
*_{i=1}^{m} a_{i}=a_{1} * a_{2} * \cdots * a_{m} .
$$

For each $a \in[0,1]$, the sequence $\left\{*^{m} a\right\}_{m=1}^{\infty}$ is defined inductively by $*^{1} a=a$ and $*^{m+1} a=\left(*^{m} a\right) * a$ for all $m \geqslant 1$. 
Remark 2.4 ([12]). If $m, n \in \mathbb{N}$, then $*^{m}\left(*^{n} a\right)=*^{m n} a$ for all $a \in \mathbb{I}$.

Definition 2.5 ([5]). A t-norm is said to be of $H$-type if the sequence $\left\{*^{m} a\right\}_{m=1}^{\infty}$ is equicontinuous at $a=1$, i.e., for all $\varepsilon \in(0,1)$, there exists $\eta \in(0,1)$ such that if $a \in(1-\eta, 1]$, then $*^{m} a>1-\varepsilon$ for all $m \in \mathbb{N}$.

Definition 2.6 ([10]). A triplet $(\mathrm{X}, \mathrm{F}, *)$ is called a Menger probabilistic metric space (for short, Menger space) if $\mathrm{X}$ is a nonempty set, $*$ is a t-norm, and $\mathrm{F}$ is a mapping from $\mathrm{X} \times \mathrm{X}$ into $\mathbb{D}^{+}$satisfying the following conditions (for $x, y \in X$, we denote $F(x, y)$ by $F_{x, y}$ ):

- $F_{x, y}(t)=H(t)$ for all $t \in \mathbb{R}$ if and only if $x=y$;

- $F_{x, y}(t)=F_{y, x}(t)$ for all $t \in \mathbb{R}$;

- $F_{x, y}(t+s) \geqslant F_{x, z}(t) * F_{z, y}(s)$ for all $x, y, z \in X$ and $t, s>0$.

We opt the following notation from [3, Lemma 2.6]: a sequence $\left\{x_{n}\right\}$ in $X$ is said to be $\mathscr{T}$-convergent to $x \in X$ (we write $x_{n} \stackrel{\mathscr{T}}{\rightarrow} x$ or $\lim _{n \rightarrow \infty} x_{n}=x$ ) if $\lim _{n \rightarrow \infty} F_{x_{n}, x}(t)=1$ for all $t>0 ;\left\{x_{n}\right\}$ is called a $\mathscr{T}$-Cauchy sequence if for any given $\varepsilon>0$ and $\lambda \in(0,1]$, there exists $N=N(\varepsilon, \lambda)$ such that $F_{x_{n}, x_{m}}(\varepsilon)>1-\lambda$, whenever $n, m \geqslant N ;(X, F, *)$ is said to be complete, if each $\mathscr{T}$-Cauchy sequence in $X$ is $\mathscr{T}$-convergent to some point in $X$.

Lemma 2.7 ([4]). Let $(\mathrm{X}, \mathrm{d})$ be a metric space. Define a mapping $\mathrm{F}: \mathrm{X} \times \mathrm{X} \rightarrow \mathscr{D}^{+}$by

$$
\mathrm{F}(\mathrm{x}, \mathrm{y})(\mathrm{t})=\mathrm{F}_{\mathrm{x}, \mathrm{y}}(\mathrm{t})= \begin{cases}0, & \text { if } \mathrm{t} \leqslant 0 \text { or } \mathrm{d}(\mathrm{x}, \mathrm{y})>\mathrm{t}>0, \\ 1, & \text { if } \mathrm{d}(\mathrm{x}, \mathrm{y}) \leqslant \mathrm{t}(\mathrm{t}>0)\end{cases}
$$

for $\mathrm{x}, \mathrm{y} \in \mathrm{X}$. Then $(\mathrm{X}, \mathrm{F}, \mathrm{min})$ is a Menger space. It is called the induced Menger space by $(\mathrm{X}, \mathrm{d})$ and it is complete iff $(\mathrm{X}, \mathrm{d})$ is complete.

In the next section, we also use the following definitions.

Definition 2.8 ([1]). We will say that the maps $f, g: X \rightarrow X$ are weakly compatible (or the pair $(f, g)$ is $w$-compatible) if $f g x=g f x$ for all $x \in X$ such that $f x=g x$.

Definition 2.9 ([12]). Given $f, g: X \rightarrow X$, a point $x \in X$ is said to be:

- fixed point of $f$ if $f x=x$;

- coincidence point of $f$ and $g$ if $f x=g x$;

- common fixed point of $f$ and $g$ if $f x=g x=x$.

Definition 2.10 ([4]). A function $\varphi: \mathbb{R}^{+} \rightarrow \mathbb{R}^{+}$is said to be right-locally monotone if for each $t \in[0, \infty)$ there exists $\delta>0$ such that $\varphi$ is monotone on $[t, t+\delta)$.

Definition 2.11 ([4]). A function $f$ on $\mathbb{R}$ is said to be piecewise monotone if there exists a partition of $[0, \infty): 0=x_{0}<x_{1}<\cdots<x_{n-1}<x_{n}=\infty$ such that $f$ is monotonic on each subinterval $\left[x_{i-1}, x_{i}\right)$ $(i=1, \cdots, n)$. These subintervals are called monotone subintervals of $f$.

\section{Main results}

In this section, we state and prove some new common fixed point theorems for weakly compatible mappings in Menger spaces by using the CLRg property and the condition (b).

Now we introduce the CLRg property in Menger spaces as follows.

Definition 3.1. Let $(X, F, *)$ be a Menger space. Two mappings $f, g: X \rightarrow X$ are said to have the CLRg property if there exists a sequence $\left\{x_{\mathrm{n}}\right\} \subseteq X$ and a point $z \in X$ such that $\mathrm{f} x_{\mathrm{n}} \stackrel{\mathscr{T}}{\rightarrow} \mathrm{gz}$ and $\mathrm{gx} \mathrm{x}_{\mathrm{n}} \stackrel{\mathscr{T}}{\rightarrow} \mathrm{gz}$ as $\mathrm{n} \rightarrow \infty$. 
Theorem 3.2. Let $(\mathrm{X}, \mathrm{F}, *)$ be a Menger space with a continuous $\mathrm{t}$-norm $*$ and let $\mathrm{f}, \mathrm{g}: \mathrm{X} \rightarrow \mathrm{X}$ be mappings having the CLRg property. Assume that $\psi: \mathbb{I} \rightarrow \mathbb{I}$ is a continuous and non-decreasing function and satisfies $\psi(1)=1$. If there exist $\mathrm{N} \in \mathbb{N}$ and $\varphi: \mathbb{R}^{+} \rightarrow \mathbb{R}^{+}$such that for each $\mathrm{t}>0$ there exists $\mathrm{r} \geqslant \mathrm{t}$ verifying $\varphi(\mathrm{r}) \leqslant \mathrm{t}$ and

$$
\mathrm{F}_{\mathrm{fx}, \mathrm{fy}}(\varphi(\mathrm{t})) \geqslant \psi\left(*^{N} \mathrm{~F}_{\mathrm{gx}, \mathrm{gy}}(\mathrm{t})\right) \text { for all } \mathrm{x}, \mathrm{y} \in \mathrm{X},
$$

then $f$ and $g$ have a coincidence point.

Proof. Since $*, \psi$, and $\mathrm{F}_{x, y}(\mathrm{t})$ are non-decreasing, it follows from (3.1) that

$$
\mathrm{F}_{f x_{n}, f z}(t) \geqslant F_{f x_{n}, f z}(\varphi(r)) \geqslant \psi\left(*^{N} F_{g x_{n}, g z}(r)\right) \geqslant \psi\left(*^{N} F_{g x_{n}, g z}(t)\right), \quad \forall t>0
$$

As $f$ and $g$ have the CLRg property, there exists a sequence $\left\{x_{n}\right\} \subseteq X$ and a point $z \in X$ such that $f x_{n} \stackrel{\mathscr{T}}{\rightarrow} g z$ and $g x_{n} \stackrel{\mathscr{T}}{\rightarrow} g z$ as $\mathrm{n} \rightarrow \infty$. That is $\lim _{\mathrm{n} \rightarrow \infty} \mathrm{F}_{\mathrm{g} x_{\mathrm{n}}, \mathrm{gz}}(\mathrm{t})=1$ for all $\mathrm{t}>0$. By the continuity of $*$ and $\psi$, we have for all $t>0$,

$$
\lim _{n \rightarrow \infty} F_{f x_{n}, f z}(t) \geqslant \psi\left(*^{N}\left(\lim _{n \rightarrow \infty} F_{g x_{n}, g z}(t)\right)\right)=\psi\left(*^{N} 1\right)=1 .
$$

Therefore, $f x_{n} \stackrel{\mathscr{T}}{\rightarrow} f z$ as $n \rightarrow \infty$. By the uniqueness of the limit, we deduce that $f z=g z$ and $z$ is a coincidence point of $f$ and $g$.

In the following corollary, $\varphi$ is a special case of the $\varphi$ used in Theorem 3.2.

Corollary 3.3. Let $(\mathrm{X}, \mathrm{F}, *), \psi$, $\mathrm{f}$ and $\mathrm{g}$ be the same as in Theorem 3.2. If there exist $\mathrm{N} \in \mathbb{N}$ and $\varphi:(0, \infty) \rightarrow$ $(0, \infty)$ such that $\varphi(t) \leqslant t$ and the condition (3.1) satisfied, then $f$ and $g$ have a coincidence point.

Proposition 3.4. Let $(\mathrm{X}, \mathrm{F}, *)$ be a Menger space. If there exist $\varphi: \mathbb{R}^{+} \rightarrow \mathbb{R}^{+}$and $\mathrm{N} \in \mathbb{N}$ such that

$$
\mathrm{F}_{\mathrm{fx}, \mathrm{fy}}(\varphi(\mathrm{t})) \geqslant *^{N} \mathrm{~F}_{g x, g y}(\mathrm{t}) \text { for all } \mathrm{x}, \mathrm{y} \in \mathrm{X} \text { and } \mathrm{t}>0,
$$

where $\mathrm{f}$ and $\mathrm{g}$ are two self-mappings from $\mathrm{X}$ to $\mathrm{X}$, then $\varphi^{\mathrm{n}}(\mathrm{t})>0$ for all $\mathrm{n} \in \mathbb{N}$ and $\mathrm{t}>0$.

Proof. If there exists some $t_{0}>0$ such that $\varphi\left(t_{0}\right)=0$, it follows from (3.2) that

$$
0=\mathrm{F}_{\mathrm{fx}, \mathrm{fx}}\left(\varphi\left(\mathrm{t}_{0}\right)\right) \geqslant * *^{\mathrm{N}} \mathrm{F}_{\mathrm{gx}, \mathrm{gx}}\left(\mathrm{t}_{0}\right)=*^{\mathrm{N}} 1=1,
$$

which is a contraction. So, $\varphi(t)>0$ for all $t>0$, which implies that $\varphi^{\mathfrak{n}}(t)>0$ for all $n \in \mathbb{N}$ and $t>0$.

Theorem 3.5. Let $(X, F, *)$ be a Menger space such that $*$ is a continuous $\mathrm{t}$-norm of H-type and let $\mathrm{f}, \mathrm{g}: \mathrm{X} \rightarrow \mathrm{X}$ be weakly compatible mappings having the CLRg property. If there exist $\varphi \in \Phi_{w}$ and $\mathrm{N} \in \mathbb{N}$ verifying the condition (3.2), then $\mathrm{f}$ and $\mathrm{g}$ have a unique common fixed point.

Proof. As $\varphi \in \Phi_{w}$, by Lemma 2.2, for each $t>0$ there exists $r \geqslant t$ such that $\varphi(r)<t$. Since $f$ and $g$ have the CLRg property, taking $\psi(t)=t$ in Theorem 3.2, there exists $z \in X$ such that $f z=g z$. For brevity, we denote $w=f z=g z$.

We shall prove that $w$ is the unique common fixed point of $f$ and $g$. Since $f$ and $g$ are weakly compatible mappings, we have $f w=f g z=g f z=g w$. We claim that

$$
F_{f w, w}\left(\varphi^{n}(t)\right) \geqslant * *^{N^{n}} F_{f w, w}(t) \text { for all } t>0 \text { and } n \in \mathbb{N} \text {. }
$$

That is obvious for $n=1$. In fact, by (3.2), we get for all $t>0$,

$$
\mathrm{F}_{\mathrm{f} w, w}(\varphi(\mathrm{t}))=\mathrm{F}_{\mathrm{f} w, f z}(\varphi(\mathrm{t})) \geqslant *{ }^{N} \mathrm{~F}_{g w, g z}(\mathrm{t})=*^{N} \mathrm{~F}_{f w, w}(\mathrm{t}) .
$$

Suppose that (3.3) holds for some $k \in \mathbb{N}$. Using Remark 2.4, Proposition 3.4, and (3.2), we have for all $\mathrm{t}>0$,

$$
\mathrm{F}_{\mathrm{f} w, w}\left(\varphi^{\mathrm{k}+1}(\mathrm{t})\right)=\mathrm{F}_{\mathrm{f} w, \mathrm{fz}}\left(\varphi\left(\varphi^{\mathrm{k}}(\mathrm{t})\right)\right) \geqslant *^{\mathrm{N}} \mathrm{F}_{g w, g z}\left(\varphi^{\mathrm{k}}(\mathrm{t})\right)
$$




$$
=*^{N} F_{f w, w}\left(\varphi^{k}(t)\right) \geqslant *^{N}\left(*^{N^{k}} F_{f w, w}(t)\right)=*^{N^{k+1}} F_{f w, w}(t),
$$

which completes the induction. Hence (3.3) holds.

Since $*$ is a t-norm of $\mathrm{H}$-type, for any $\varepsilon \in(0,1)$ there exists $\eta \in(0,1)$ such that

$$
\text { if } a \in(1-\eta, 1] \text {, then } *^{m} a>1-\varepsilon \text { for all } m \in \mathbb{N} \text {. }
$$

Owing to $\mathrm{F}_{\mathrm{f} w, w}(\mathrm{t}) \rightarrow 1$ as $\mathrm{t} \rightarrow \infty$, there exists $\mathrm{t}_{1}>0$ such that $\mathrm{F}_{\mathrm{f} w, w}\left(\mathrm{t}_{1}\right)>1-\eta$. Since $\varphi \in \Phi_{w}$, there exists $t_{2} \geqslant t_{1}$ such that $\lim _{n \rightarrow \infty} \varphi^{n}\left(t_{2}\right)=0$. Thus, for any $t>0$, there exists $n_{0} \in \mathbb{N}$ such that $\varphi^{n}\left(t_{2}\right)<t$ for all $n \geqslant n_{0}$. From (3.3) and (3.4), we get for all $n \geqslant n_{0}$ and $t>0$,

$$
\mathrm{F}_{\mathrm{f} w, w}(\mathrm{t}) \geqslant \mathrm{F}_{\mathrm{f} w, w}\left(\varphi^{\mathrm{n}}\left(\mathrm{t}_{2}\right)\right) \geqslant *^{N^{n}} \mathrm{~F}_{\mathrm{f} w, w}\left(\mathrm{t}_{2}\right) \geqslant *^{N^{n}} \mathrm{~F}_{\mathrm{f} w, w}\left(t_{1}\right)>1-\varepsilon,
$$

which implies that $F_{f w, w}(t)=1$ for all $t>0$. So, $f w=w$ and $w$ is a common fixed point of $f$ and $g$.

To prove the uniqueness, let $y \in X$ be another common fixed point of $f$ and $g$, that is $f y=g y=y$. On using same argument which used to establish claim (3.3), we can prove that

$$
\mathrm{F}_{w, y}\left(\varphi^{n}(t)\right) \geqslant *{ }^{N^{n}} F_{w, y}(t) \text { for all } n \in \mathbb{N} \text { and } t>0 \text {. }
$$

Since $F_{w, y}(t) \rightarrow 1$ as $t \rightarrow \infty$, there exists $t_{3}>0$ such that $F_{w, y}\left(t_{3}\right)>1-\eta$. Since $\varphi \in \Phi_{w}$, there exists $t_{4} \geqslant t_{3}$ such that $\varphi^{n}\left(t_{4}\right) \rightarrow 0$ as $n \rightarrow \infty$. So, for any $t>0$, there exists $n_{1} \in \mathbb{N}$ such that $\varphi^{n}\left(t_{4}\right)<t$ for all $n \geqslant n_{1}$. By (3.4) and (3.5), we deduce that for all $t>0$ and $n \geqslant n_{1}$,

$$
F_{w, y}(t) \geqslant F_{w, y}\left(\varphi^{n}\left(t_{4}\right)\right) \geqslant *^{N^{n}} F_{w, y}\left(t_{4}\right) \geqslant *^{N^{n}} F_{w, y}\left(t_{3}\right)>1-\varepsilon .
$$

So, $F_{w, y}(t)=1$ for all $t>0$, that is $w=y$. This completes the proof.

In order to illustrate the contributions of Theorem 3.5, we provide the following example in which other theorems in $[2,4,8]$ cannot be applied.

Example 3.6. Let $\mathrm{X}=[0,1), *=\min$, and define $\mathrm{F}: \mathrm{X} \times \mathrm{X} \rightarrow \mathscr{D}^{+}$as follows:

$$
F_{x, y}(t)= \begin{cases}0, & \text { if } t \leqslant 0 \\ 1, \quad & \text { if }|x-y|<t(t>0) \\ \frac{t}{|x-y|+t}, & \text { if }|x-y| \geqslant t(t>0)\end{cases}
$$

By the similar argument to Example in [2], $(\mathrm{X}, \mathrm{F}, *)$ is a Menger space.

Let $\varphi$ be as in Example 2.1, then $\phi \in \Phi_{w}$ but $\phi \notin \Phi$. Consider $f, g: X \rightarrow X$ defined by

$$
f x=\left\{\begin{array}{ll}
\frac{x}{1+x}, & \text { if } x \in\left[0, \frac{1}{2}\right), \\
0, & \text { if } x \in\left[\frac{1}{2}, 1\right) ;
\end{array} \quad g x= \begin{cases}2 x, & \text { if } x \in\left[0, \frac{1}{2}\right), \\
0, & \text { if } x \in\left[\frac{1}{2}, 1\right) .\end{cases}\right.
$$

It is easy to see that $z=0$ and $z \in\left[\frac{1}{2}, 1\right)$ are all coincidence points of $f$ and $g$. If $z \in\left[\frac{1}{2}, 1\right)$, then $f g(z)=g f(z)$. Furthermore, as $f g(0)=g f(0), f$ and $g$ are weakly compatible. Consider a sequence $\left\{\frac{1}{3^{n}}\right\}_{\mathfrak{n} \in \mathbb{N}}$ and a point $z=0$. For any $t>0$, there exists $n_{2} \in \mathbb{N}$ such that $\frac{1}{3^{n}+1}<t$ for all $n \geqslant n_{2}$. Therefore, for any $t>0$ and $\varepsilon \in(0,1)$, we have that $F_{f \frac{1}{3^{n}}, g 0}(t)=1>1-\varepsilon$ for all $n \geqslant n_{2}$, which implies that $f_{\frac{1}{3^{n}}} \stackrel{\mathscr{T}}{\rightarrow} g 0$. Similarly, we get $\mathrm{g} \frac{1}{3^{n}} \stackrel{\mathscr{T}}{\rightarrow} \mathrm{g} 0$. Therefore, $f$ and $\mathrm{g}$ have the CLRg property in $(X, F, *)$.

Next, we shall prove that (3.2) holds for $N=1$. If $x, y \in\left[\frac{1}{2}, 1\right)$, then $F_{f x, f y}(\varphi(t))=1$ and so (3.2) trivially holds for $N=1$. Suppose that $x \in\left[0, \frac{1}{2}\right)$ and $y \in\left[\frac{1}{2}, 1\right)$ (the case $y \in\left[0, \frac{1}{2}\right)$ and $x \in\left[\frac{1}{2}, 1\right)$ is treated similarly). Then $|f x-f y|=\frac{x}{1+x}$ and $|g x-g y|=2 x$. If $\frac{x}{1+x}<\varphi(t)$, then $F_{f x, f y}(\varphi(t))=1$ and so (3.2) holds for $N=1$. Note that $\varphi(t) \geqslant \frac{t}{1+t}$ for all $t>0$. If $\frac{x}{1+x} \geqslant \varphi(t)$, then $x \geqslant t$ because the function $f(u)=\frac{u}{1+u}$ is strictly increasing on $[0, \infty)$. From (3.6), we get for all $t>0$,

$$
\mathrm{F}_{\mathrm{fx}, \mathrm{fy}}(\varphi(\mathrm{t}))=\frac{\varphi(\mathrm{t})}{\frac{\mathrm{x}}{1+x}+\varphi(\mathrm{t})} \geqslant \frac{\frac{\mathrm{t}}{1+\mathrm{t}}}{\frac{\mathrm{t}}{1+\mathrm{t}}+\frac{\mathrm{x}}{1+x}} \geqslant \frac{\mathrm{t}}{2 \mathrm{x}+\mathrm{t}}=\mathrm{F}_{\mathrm{gx}, \mathrm{gy}}(\mathrm{t}),
$$


i.e., (3.2) holds for $N=1$.

Suppose that $x, y \in\left[0, \frac{1}{2}\right)$. Notice that

$$
|f x-f y|=\frac{|x-y|}{1+x+y+x y}=\frac{|x-y|}{1+|x-y|+2 \min \{x, y\}+x y} \leqslant \frac{|x-y|}{1+|x-y|} .
$$

If $|f x-f y|<\varphi(t)$, then $F_{f x, f y}(\varphi(t))=1$ and so (3.2) holds for $N=1$. If $|f x-f y| \geqslant \varphi(t)$, by (3.7), then $\frac{t}{1+t} \leqslant \frac{|x-y|}{1+|x-y|}$, which implies that $|x-y| \geqslant t$. It follows from (3.6) that $F_{g x, g y}(t)=\frac{t}{2|x-y|+t}$. Therefore, for all $t>0$,

$$
F_{f x, f y}(\varphi(t))=\frac{\varphi(t)}{|f x-f y|+\varphi(t)} \geqslant \frac{\frac{t}{1+t}}{\frac{t}{1+t}+\frac{|x-y|}{1+|x-y|}} \geqslant \frac{t}{2|x-y|+t}=F_{g x, g y}(t),
$$

i.e., (3.2) holds for $\mathrm{N}=1$.

This shows that all the conditions of Theorem 3.5 are satisfied. Thus, $f$ and $g$ have a unique common fixed point, which is $z=0$. However, other results of papers $[2,4,8]$ can not be applied because $(\mathrm{X}, \mathrm{F}, *)$ is not complete. In fact, from (3.6), we know that for any $\varepsilon>0,|x-y|<\varepsilon$ iff $F_{x, y}(\varepsilon)=1$. Thus, it is easy to prove that $\left\{x_{n}\right\}$ is a Cauchy sequence in $(X, d)$ iff $\left\{x_{n}\right\}$ is a $\mathscr{T}$-Cauchy sequence in $(X, F, *)$, where $\mathrm{d}(x, y)=|x-y|$. And $x_{n} \rightarrow x$ in $(X, d)$ iff $x_{n} \stackrel{\mathscr{T}}{\rightarrow} x$ in $(X, F, *)$. So, $(X, F, *)$ is complete iff $(X, d)$ is complete. Since $(X, d)$ is not complete, we deduce that $(X, F, *)$ is also not complete.

Corollary 3.7. Let $(\mathrm{X}, \mathrm{F}, *), \mathrm{f}$, and $\mathrm{g}$ be the same as in Theorem 3.5. If there exists $\mathrm{N} \in \mathbb{N}$ such that

$$
\mathrm{F}_{\mathrm{fx}, \mathrm{fy}}(\alpha(\mathrm{t}) \mathrm{t}) \geqslant *^{N} \mathrm{~F}_{\mathrm{gx}, \mathrm{gy}}(\mathrm{t}) \text { for all } \mathrm{x}, \mathrm{y} \in \mathrm{X} \text { and } \mathrm{t}>0,
$$

where $\alpha: \mathbb{R}^{+} \rightarrow[0,1)$ is a piecewise monotone function, then $\mathrm{f}$ and $\mathrm{g}$ have a unique common fixed point.

Proof. Denote $\varphi(t)=\alpha(t) t$. From the proof of Theorem 3.3 in [4], $\varphi \in \Phi_{w}$. It follows from (3.8) that (3.2) holds. Therefore, we obtain the conclusion of this corollary by Theorem 3.5.

From the proof of Theorem 3.2 and Corollary 3.2 in [4], we know that $\varphi \in \Phi_{w}$ in the following results. So, we obtain the following results by Theorem 3.5 immediately.

Corollary 3.8. Let $(\mathrm{X}, \mathrm{F}, *), \mathrm{f}$, and $\mathrm{g}$ be the same as in Theorem 3.5. Let $\varphi: \mathbb{R}^{+} \rightarrow \mathbb{R}^{+}$be a right-locally monotone function satisfying the condition (CBW). If there exists $\mathrm{N} \in \mathbb{N}$ verifying the condition (3.2), then $\mathrm{f}$ and $\mathrm{g}$ have a unique common fixed point.

Corollary 3.9. Let $(\mathrm{X}, \mathrm{F}, *), \mathrm{f}$, and $\mathrm{g}$ be the same as in Theorem 3.5. Let $\varphi: \mathbb{R}^{+} \rightarrow \mathbb{R}^{+}$be a function satisfying the condition $(B W): \varphi(t)<t$ and $\lim \sup _{r \rightarrow t^{+}} \varphi(r)<t$ for each $t>0$. If there exists $\mathrm{N} \in \mathbb{N}$ verifying the condition (3.2), then $\mathrm{f}$ and $\mathrm{g}$ have a unique common fixed point.

Taking $\mathrm{g}=\mathrm{I}_{\mathrm{X}}$ (the identity mapping on $\mathrm{X}$ ) in Theorem 3.5 and Corollaries 3.7-3.9, we obtain the following Corollaries 3.10-3.14.

Corollary 3.10. Let $(\mathrm{X}, \mathrm{F}, *)$ be a Menger space such that $*$ is a continuous $\mathrm{t}$-norm of $\mathrm{H}$-type and let $\mathrm{f}: \mathrm{X} \rightarrow \mathrm{X}$ be a mapping such that there exists a sequence $\left\{x_{n}\right\} \subseteq X$ verifying $\lim _{n \rightarrow \infty} f x_{n}=\lim _{n \rightarrow \infty} x_{n}$. If there exist $\varphi \in \Phi_{w}$ and $\mathrm{N} \in \mathbb{N}$ satisfying

$$
\mathrm{F}_{\mathrm{fx}, \mathrm{fy}}(\varphi(\mathrm{t})) \geqslant *^{N} \mathrm{~F}_{\mathrm{x}, \mathrm{y}}(\mathrm{t}) \text { for all } \mathrm{x}, \mathrm{y} \in \mathrm{X} \text { and } \mathrm{t}>0,
$$

then $\mathrm{f}$ has a unique fixed point $z \in \mathrm{X}$, and $\left\{\mathrm{f} \mathrm{x}_{\mathrm{n}}\right\}$ converges to $z$.

In order to illustrate the contributions of Corollary 3.10, we provide the following example in which other theorems in $[2,4,8]$ cannot be applied. 
Example 3.11. Let $(X, F, *), f$, and $\varphi$ be the same as in Example 3.6. Consider a sequence $\left\{\frac{1}{3^{n}}\right\}_{\mathfrak{n} \in \mathbb{N}}$ and a point $z=0$. By the similar argument to Example 3.6, all conditions of Corollary 3.10 are satisfied for $\mathrm{N}=1$. Thus, $\mathrm{f}$ has a unique fixed point $z=0$.

Corollary 3.12. Let $(\mathrm{X}, \mathrm{F}, *)$ and $\mathrm{f}$ be the same as in Corollary 3.10. If there exists $\mathrm{N} \in \mathbb{N}$ such that

$$
\mathrm{F}_{\mathrm{fx}, \mathrm{fy}}(\alpha(\mathrm{t}) \mathrm{t}) \geqslant *^{N} \mathrm{~F}_{\mathrm{x}, \mathrm{y}}(\mathrm{t}) \text { for all } \mathrm{x}, \mathrm{y} \in \mathrm{X} \text { and } \mathrm{t}>0,
$$

where $\alpha: \mathbb{R}^{+} \rightarrow[0,1)$ is a piecewise monotone function, then $\mathrm{f}$ has a unique fixed point $z \in X$, and $\left\{\mathrm{f} x_{n}\right\}$ converges to $z$.

Corollary 3.13. Let $(\mathrm{X}, \mathrm{F}, *)$ and $\mathrm{f}$ be the same as in Corollary 3.10. Let $\varphi: \mathbb{R}^{+} \rightarrow \mathbb{R}^{+}$be a right-locally monotone function satisfying the condition (CBW). If there exists $\mathrm{N} \in \mathbb{N}$ verifying the condition (3.9), then $\mathrm{f}$ has a unique fixed point $z \in X$, and $\left\{\mathrm{fx}_{\mathrm{n}}\right\}$ converges to $z$.

Corollary 3.14. Let $(\mathrm{X}, \mathrm{F}, *)$ and $\mathrm{f}$ be the same as in Corollary 3.10. Let $\varphi: \mathbb{R}^{+} \rightarrow \mathbb{R}^{+}$be a function satisfying the condition (BW). If there exists $\mathrm{N} \in \mathbb{N}$ verifying the condition (3.9), then $\mathrm{f}$ has a unique fixed point $z \in X$, and $\left\{f x_{n}\right\}$ converges to $z$.

Using Theorem 3.5, we obtain the following fixed point result in usual metric spaces.

Corollary 3.15. Let $(\mathrm{X}, \mathrm{d})$ be a metric space and let $\mathrm{f}, \mathrm{g}: \mathrm{X} \rightarrow \mathrm{X}$ be weakly compatible mappings having the CLRg property. Assume that $\varphi: \mathbb{R}^{+} \rightarrow \mathbb{R}^{+}$is non-decreasing function in $\Phi_{w}$ and satisfies $\varphi(t)>0$ for all $\mathrm{t}>0$. If

$$
d(f x, f y) \leqslant \varphi(d(g x, g y)) \text { for all } x, y \in X
$$

then $\mathrm{f}$ and $\mathrm{g}$ have a unique common fixed point.

Proof. Consider a mapping $\mathrm{F}: \mathrm{X} \times \mathrm{X} \rightarrow \mathscr{D}^{+}$defined by (2.1). Since $(\mathrm{X}, \mathrm{d})$ is a metric space, by Lemma 2.7, we find that $(X, F, \min )$ is a Menger space. As $f$ and $g$ have the CLRg property in $(X, d)$, there exists a sequence $\left\{x_{n}\right\} \subseteq X$ and a point $z \in X$ such that $f x_{n} \rightarrow g z$ and $g x_{n} \rightarrow g z$ as $n \rightarrow \infty$. So, for any $t>0$, there exists $n_{3} \in \mathbb{N}$ such that $d\left(f x_{n}, g z\right) \leqslant t$ for all $n \geqslant n_{3}$. It follows from (2.1) that $F_{f x_{n}, g z}(t)=1$ for all $\mathrm{n} \geqslant \mathrm{n}_{3}$, which implies that $\mathrm{f} x_{\mathrm{n}} \stackrel{\mathscr{T}}{\rightarrow} \mathrm{g} z$ in $(X, F, \min )$. Similarly, we get $\mathrm{g} x_{\mathrm{n}} \stackrel{\mathscr{T}}{\rightarrow} \mathrm{g} z$ in $(X, \mathrm{~F}, \min )$. Therefore, $f$ and $g$ have the CLRg property in $(X, F, \min )$.

Next, we shall prove that the condition (3.10) implies (3.2). If $F_{g x, g y}(t)=0$ for all $t>0$, then (3.2) trivially holds. If $\mathrm{F}_{g x, g y}(t)=1$, then by $(2.1)$ we find that $\mathrm{d}(\mathrm{gx}, \mathrm{gy}) \leqslant \mathrm{t}$. Since $\varphi$ is non-decreasing, it follows from (3.10) that $d(f x, f y) \leqslant \varphi(d(g x, g y)) \leqslant \varphi(t)$. Since $\varphi(t)>0$ for all $t>0$, we have $F_{f x, f y}(\varphi(t))=1=F_{g x, g y}(t)$. Hence (3.2) holds. Using Theorem 3.5, we deduce that $f$ and $g$ have a unique common fixed point.

Remark 3.16. Corollaries 3.10-3.15 are generalizations and improvements of Theorems 3.1, 3.3, 3.2 and Corollaries 3.2 and 3.3 in [4], respectively. Indeed, if $N=1$ in (3.9), then $f: X \rightarrow X$ is a probabilistic $\varphi$-contraction. Our results show that the completeness condition of $(X, F, *)$ and $(X, d)$ in $[3$, Theorems 3.1-3.3 and Corollaries 3.2 and 3.3] is not necessary. Note also that if $N=1$ in our results, then the continuity of $*$ is unnecessary.

\section{Acknowledgment}

This work is supported by the Natural Science Foundation of Jiangsu Province under Grant (No. 16KJB110022) and (No. 16KJD110006), and National Science Foundation of China (No. 11671287). 


\section{References}

[1] M. Abbas, M. Ali Khan, S. Radenovic, Common coupled fixed point theorems in cone metric spaces for w-compatible mappings, Appl. Math. Comput., 217 (2010), 195-202. 2.8

[2] L. Cirić, Solving the Banach fixed point principle for nonlinear contractions in probabilistic metric spaces, Nonlinear Anal., 72 (2010), 2009-2018. 1, 3, 3.6, 3.6, 3

[3] J.-X. Fang, Common fixed point theorems of compatible and weakly compatible maps in Menger spaces, Nonlinear Anal., 71 (2009), 1833-1843. 1

[4] J.-X. Fang, On $\varphi$-contractions in probabilistic and fuzzy metric spaces, Fuzzy Sets and Systems, 267 (2015), 86-99. 1, 2.1, 2.2, 2.7, 2.10, 2.11, 3, 3.6, 3, 3, 3.16

[5] O. Hadžić, E. Pap, Fixed Point Theory in Probabilistic Metric Spaces, Kluwer Academic Publishers, Dordrecht, (2001). 2.5

[6] X.-Q. Hu, Common coupled fixed point theorems for contractive mappings in fuzzy metric spaces, Fixed Point Theory Appl., 2011 (2011), 14 pages. 1

[7] X.-Q. Hu, M.-X. Zheng, B. Damjanović, X.-F. Shao, Common coupled fixed point theorems for weakly compatible mappings in fuzzy metric spaces, Fixed Point Theory Appl., 2013 (2013), 11 pages. 1

[8] J. Jachymski, On probabilistic ф-contractions on Menger spaces, Nonlinear Anal., 73 (2010), 2199-2203. 1, 3, 3.6, 3

[9] M. Jain, K. Tas, S. Kumar, N. Gupta, Coupled fixed point theorems for a pair of weakly compatible maps along with CLRg property in fuzzy metric spaces, J. Appl. Math., 2012 (2012), 13 pages. 1

[10] K. Menger, Statistical metrics, Proc. Natl. Acad. Sci., 1942 (1942), 535-537. 2.6

[11] D. O'Regan, R. Saadati, Nonlinear contraction theorems in probabilistic spaces, Appl. Math. Comput., 195 (2008), 86-93. 1

[12] A.-F. Roldn-Lpez-de-Hierro, W. Sintunavarat, Common fixed point theorems in fuzzy metric spaces using the CLRg property, Fuzzy Sets and Systems, 282 (2016), 131-142. 1, 2.4, 2.9

[13] B. Schweizer, A. Sklar, Probabilistic Metric Spaces, Dover Publications, New York, (2005). 2.3

[14] V. M. Sehgal, A. T. Bharucha-Reid, Fixed points of contraction mappings on probabilistic metric spaces, Math. Systems Theory, 6 (1972), 97-102. 1

[15] W. Sintunavarat, P. Kumam, Common fixed point theorems for a pair of weakly compatible mappings in fuzzy metric spaces, J. Appl. Math., 2011 (2011), 14 pages. 1 